\title{
Tangible Cubes as Programming Objects
}

\author{
Andrew C Smith \\ Meraka Institute, CSIR \\ South Africa \\ acsmith@csir.co.za
}

\begin{abstract}
In this paper, we describe early results in the development of an alternative programming method which uses large, three-dimensional acrylic blocks to construct programming sequences. Computerilliterate children can create a symbolic program to control a humanoid robot toy. We describe the physical design of the blocks, problems encountered, comments made by primary school participants, and make recommendations for further development.
\end{abstract}

\section{Introduction}

The writings by people such as Neil Gershenfeld [1, p140], who in 1999 authored the book "When things start to think" are inspiring. In it he explores the idea of "moving" computing out of the conventional computers into everyday objects. This triggered in us the idea of using everyday objects to write computer programmes. Instead of arranging words and symbols on a computer screen, what would happen if we arranged physical objects instead? With low-cost processors and radio frequency identification components so readily available, this idea did not seem so far-fetched. Thus was born the idea of programming using physical cubes.

Our eventual aim is to introduce pre-school children to the logical thinking processes required for programming. We hypothesize that if children are introduced to these concepts in a playful manner, they will find it simple to master the formal programming environments when exposed to them later. This paper does not test this hypothesis however, it simply reports on the preliminary work done that will form the basis of more extensive research in the future. Our research addresses alternative programming environments, as alternatives to the currently dominant use of keyboard and display screen. We also want to encourage physical movement amongst children in an era when adults complain of excessive wrist strain when spending extended time in front of a computer. The question we ask is whether it is possible to incorporate logical thinking/programming in the natural play of young children, typically in the age group of 3-5 years.

The system we describe represents the currents state of our research and development. It allows for sequential, linear execution of instructions, and it is stateless (no awareness of its own state). The building elements contain magnetic parts and not electronic, as opposed to "electronic blocks" described in other research papers [5].

Traditional programming environments require familiarity of at least the computer keyboard, where the letters and numbers are located. Then there is the knowledge required for "saving" the code in a file, and compiling the code to an executable and downloading it to the target (the robot in this instance). Alternatively, an interpreted language can be used, either text-based or graphical (such as Lego Mindstorms RCX(C) programming environment). Still, the child needs to control the mouse to move the programming elements around. It is unfortunate that knowledge of the computer environment is required to do basic programming. Perhaps, one day, being computer literate will no longer be a prerequisite to being called a programmer.

We are researching an approach which sidesteps this requirement, manipulating physical objects as the code itself. We envisage that preschool children would be able to write code using large, but lightweight, three-dimensional objects as representations of the instructions. Examples of such objects currently exist in the playground, albeit not yet suitable for use as programming objects because the required electronic circuitry has not yet been embedded inside the objects. It is a pity that we tend to reduce the size of physical interfaces to computers as technology improves. Children have abundant energy and would probably prefer to be physically active and use whole-body movements to construct a programme, as apposed to be seated at a mall computer keyboard and screen. The programming 
language still needs to be defined properly and refined.

\section{Technical description}

The programming environment consists of a number of acrylic cubes, each embedded with permanent magnets. This approach differs from those that require electrical contact between blocks [5][6]. In this implementation the cubes are placed on acrylic "trays" which contain sensing circuitry and are connected to a central controller. The controller senses which cube is positioned in each allowable position and sends appropriate commands to a toy robot. Allowed positions are currently limited to those indicated by the trays. Magnetic door/window alarm switches are used in the system described here. This is a combination of a bar magnet and a reed switch. Both are encapsulated in plastic. They are used in the cubes and trays to identify and sense the cubes. The magnets are mounted on the inside surface of the cube bottom. This design allows for $2^{\wedge} 5-1$ combinations, five potential locations exist for the magnets on the cube surface. We encode the function with a binary representation of the function number. The spacing between magnets is such that a magnet will not affect the adjacent sensors in the tray. The cubes are colour coded, with two cubes of each colour.

Once the system has been activated, code interpretation starts at the first tray and proceeds sequentially along the trays until they have all been queried. There are two ways to proceed. The first mode of execution is to wait for a cube to be placed on a tray. Execution is then paused at the next tray position until a cube is placed there. Execution continues in this manner, pausing where there is no cube. This operation method has the advantage that the child can immediately observe the effect the cube has on the robot. The disadvantage is that coding with loops (once the syntax has been developed to support it) will be problematic. If a tray is passed over and a cube is placed on a tray more than one position away from the previous one, the system assumes that the space represents a NOP (nooperation) and immediately interprets the newly placed cube. An alternative mode of execution is to halt the execution before the first tray is interrogated, and place all the cubes in the desired positions and sequence. When the programme "design" has been completed, the halt is removed and interpretation of the complete code construction is done.

A toy robot is used to demonstrate the principle of controlling a physical object by the relative placement of physical cubes. The following six functionalities have been implemented: turn the body left/right, turn the head left/right, and move the body forward/backwards. The movements are all incremental. To achieve maximum body and head turning would require repetition of an instruction to ensure extreme movement in the selected direction. For instance, to move the head from the forwardfacing position to right-facing position requires three commands in succession. Forward and backward body movement instructions result in the internal robot motors being activated for approximately one second. This equates to a linear movement of $30 \mathrm{~cm}$.

\section{Usability study}

Many children experienced the thrill of their first ever programme composition during the workshops conducted at two science shows.

We were interested in the usability and educational value of this experiment. For this we contracted an independent company that specializes in usability evaluations. The test was complicated because the users could not be specified. The experiment was conducted as part of the science shows and was aimed at schools-going children and the public at large. Approximately 5000 children visited each science show, with groups of 20 children interacting with the blocks during a 45-minute session. The results are based on a single group of children, but the interaction was staggered in that a single child would be afforded an opportunity to interact with the blocks, while the rest of the group observed. A second child would be given an opportunity and so forth.

The study [2] shows that it was difficult for children to initially understand how the programming environment works. After some coaching by the facilitator the children realized that the cubes had to be placed on the trays. According to ISO 9241-11, the definition of usability is "the extent to which a product can be used by specified users to achieve specified goals with effectiveness, efficiency and satisfaction in a specified context of use".

We found that some children had difficulty in associating the robot movement with the block that represented the current instruction. Norman [3, p50] uses the term "gulf" to describe this separation between mental states and the physical state. This would typically happen once the robot had executed a few movements, specifically rotations. It can happen that the robot has rotated its body through 180 degrees and now faces the opposite direction to when the sequence started. Now, left and right have been "interchanged". When programming, the child would not take this into consideration. In other words, the co-ordinates of the robot are different to the coordinates of the world, but the programming 
child programmes in the word's coordinates. To the child the outcome is not what was expected. Once it was explained that the robot executes instructions from its own perspective, the children typically understood the difference in coordinates and made corrections for this in the next programming attempt. These co-ordinates are the same as those described by Papert [4, p55] for the Logo turtle. The robot faces a direction which could change as it executes commands received from the tray. Thus left can "become" right, and right can "become" left.

\section{Effectiveness}

The study shows that the symbols used on the cubes are not effective at all. They are not intuitive and required some explanation by the facilitator. The orientation was not clear either. The cube has six sides and it was not clear which side should face up when positioned correctly. The correlation between the direction indicated by the symbol and the direction the robot moved was not strong. It was clear that significant learning was required in order to master the use of the blocks.

\subsection{Efficiency}

Almost 15 minutes were required by the first child to master the use of the blocks. Subsequent children required in the order of 6 minutes to master the use of the blocks, an improvement of $60 \%$. The improvement was due to the learning that took place by observing the first child, and also working in a small group of two and three children.

\subsection{Satisfaction}

Both positive and negative feedback was received from the children. The children reported that they enjoyed the idea and challenge of controlling the robot. The negative feedback included aspects such as the size of the cubes which were too large, that the robot moved relative to its current position, and not relative to the environment.

\subsection{Comment by some children}

It was interesting that when older children (ages 10-12 years) were exposed to the programming objects presented in this paper, some children commented that the blocks were too large. They would prefer something smaller that could be used on a desk, similar to the size of Lego blocks. This is exactly the opposite of what we aimed to achieve, we were hoping that the children would be physically more active and not simply sit in one place when programming. The younger children were quite happy with the large blocks.

\section{What worked well}

We observed that the locating tabs on the trays helped position the blocks properly on the trays. So too does the writing on the front of the blocks help in identifying the orientation of the blocks, which is the same for all the blocks. In the current implementation, the symbols that indicate the functions are located on one side only, the top of the blocks. However, from our observations this did not seem to be sufficient indication on how the blocks had to be placed on the trays. Coaching was still required.

The low-cost toy robot "Jackey" was popular with the children. Its appearance and pre-recorded "voice" were appealing aspects to the children.

We found it beneficial to use large blocks as the children could easily see what the programme was supposed to do. There was no need to look at a small computer screen or jostling for a position around the screen by the spectators. At a distance of $5 \mathrm{~m}$ all the children were able to contribute to the programming activity. This is possibly a new form of programming approach: spectator-based team work, as apposed to singular- or peer-programming.

\section{Areas requiring improvement}

The trays and blocks are made out of acrylic. This material is brittle and too unforgiving, cracking when dropped. The orientation of the blocks was sometimes an issue to the participants. It was not always clear which way was front and top. The logos on the blocks did not clearly indicate the functionality. The infrared communication with the toy robot was not reliable. The modified remote controller was not reliable. It had to be replaced as it became evident that it was losing its functionality. Fewer and fewer functions remained operational as testing progressed in the laboratory. We suspect that the wiring (added in parallel with the buttons) interfered with the on-board controller circuit. The magnets in the cubes did not adhere well when we used a hot glue gun. Using an appropriate glue, or preparing the acrylic surface (by lightly sanding it), would probably improve adhesion

\section{Future}

We plan to implement more of the TURTLE TALK [4, p56] language in a future version of the system. This will include the option of adding parameters to a coding instruction, for example 
MOVE BODY LEFT, 3 TIMES. To do the same with the current implementation requires repeating the same instruction three times: MOVE BODY LEFT, MOVE BODY LEFT, MOVE BODY LEFT.

We aim to eventually develop physical blocks that will allow the programming, as opposed to simply controlling, remote objects by including the functionality of conditional code execution and programming loops. To realize this, the blocks need to be stateful (an awareness of its current state).

We also aim to develop blocks that are fully "aware" that they occupy three dimensions in space. That is, its orientation is significant and the blocks can be stacked in three dimensions. In this form, no trays will be required and the blocks communicate with each other and are aware of what other blocks surround them. The self-discovery nature of this system will be similar to routing algorithms used in computer data networks.

We anticipate that visual indicators of the communication process will be added to aid the understanding of the computation process taking place between blocks. As data packets transverse the blocks, a visual or auditory indication is made. This would show the transient nature of data packets.

We aim to have blocks that have multiple functions, depending on its orientation. With six sides to a cube, the possibility exists to have six different functions per cube. The function that the cube encodes is determined by the side that faces up. The current implementation that utilizes magnets for the function encoding can already provide this functionality, but we are aiming to have a system that can have multiple layers of cubes stacked on each other. This would probably exclude the use of magnets and trays, as in the current configuration.

We hope to investigate alternative indication mechanisms, such as using electromagnetic fields. This could typically operate at a carrier frequency of $125 \mathrm{kHz}$ and use induction loops, similar to what is in current us for Radio Frequency Identification systems.

\section{Acknowledgements}

Mpho Nkuna, an undergraduate student at the Meraka Institute implemented the controlling electronics and presented the workshops. Corne Kruger from the company Test and Data Services designed the tests and reported on the usability and educational value of the system and made recommendations for improvements. The FabLab was responsible for manufacturing most of the acrylic trays and blocks. Reinett Mafentile assembled the blocks and trays and assisted with the logistics of the various workshops. The research was funded by the South African Department of Science and Technology.

\section{References}

[1] Neil Gershenfeld, FAB: Coming revolution on your desktop - from personal computers to personal fabrication, Basic Books, 2005.

[2] Bekker, A., Kruger C., "Usability and educational review on GameBlocks and Body Ping Pong". Test report, Test and Data Services, Available from

http://playpen.icomtek.csir.co.za/ acdc/education/Science Unlimited2006/reports/ICT in Education Report 1.0.doc , 26 April 2006.

[3] Donald A. Norman, The design of everyday things.

[4] Seymour Papert, Mindstorms: Children, Computers, and Powerful Ideas, Basic Books.

[5] Wyeth, P., Purchase, H.C, "Programming without a computer: a new interface for children under eight", User Interface Conference, 2000. AUIC 2000. First Australasian, Date: 2000, Pages: $141-148$.

[6] Trung Dung Ngo, Henrik Hautop Lund, "Modular artifacts", Adaptronics group.

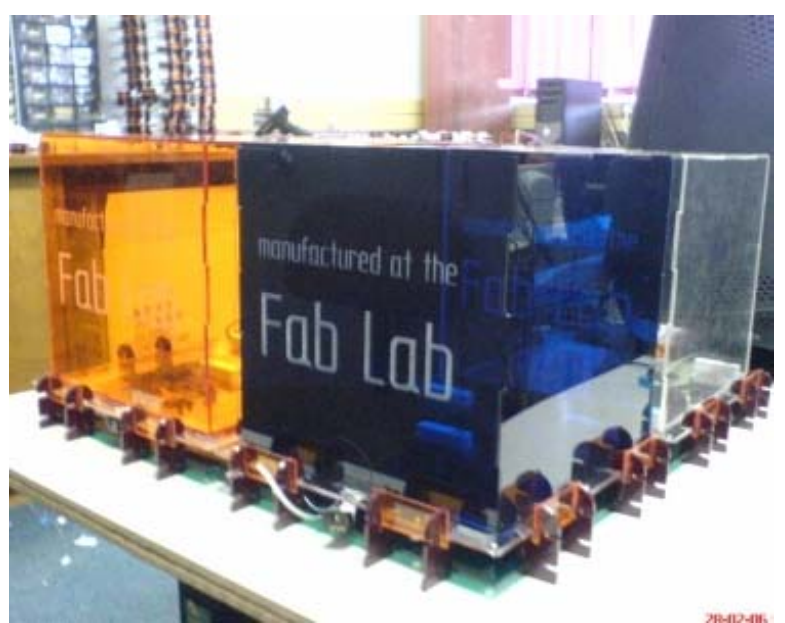

Figure 1. Acrylic cubes of various colours placed on the trays, ready for interpretation and execution. 


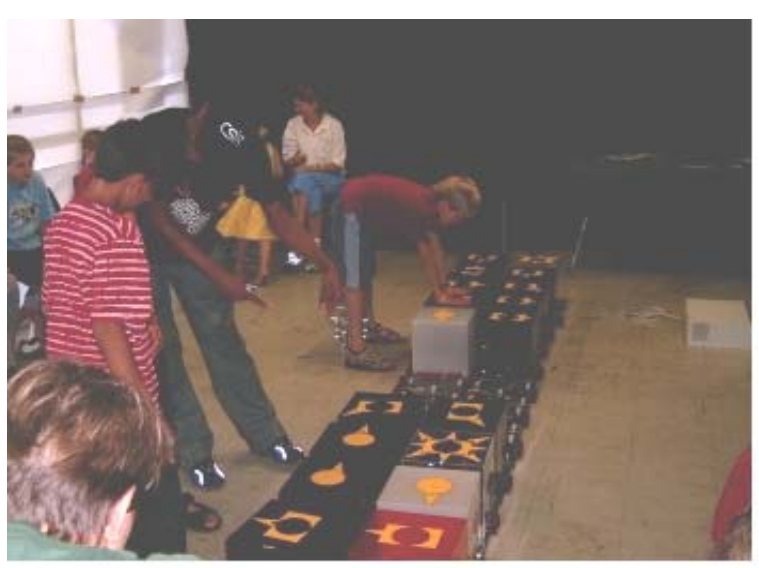

Figure 2. Children place the cubes on the trays to "code" a programme. The symbols on the cubes indicate the movement that will be executed by the toy robot.

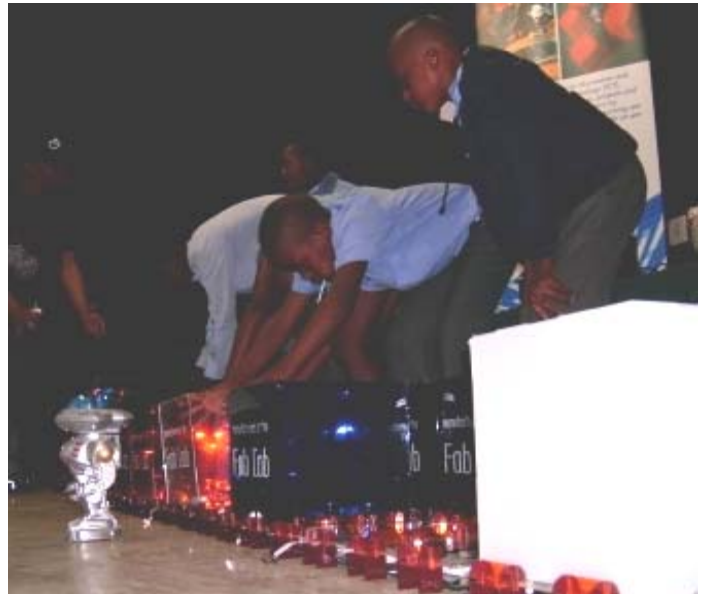

Figure 3. The toy robot executes the instructions as they are placed on the trays. 